\title{
The dyad gene is required for progression through female meiosis in
}

\section{Arabidopsis}

\author{
Imran Siddiqi1,^, Gopal Ganesh¹, Ueli Grossniklaus²,‡ and Veeraputhiran Subbiah1 \\ ${ }^{1}$ Centre for Cellular and Molecular Biology, Uppal Road, Hyderabad-500007, India \\ ${ }^{2}$ Cold Spring Harbor Laboratory, 1 Bungtown Road, Cold Spring Harbor, NY11724, USA \\ ¥Present address: Friedrich Miescher Institute, Postfach 2543, CH-4002 Basel, Switzerland \\ *Author for correspondence (e-mail: imran@ccmb.ap.nic.in) \\ Accepted 15 October; published on WWW 8 December 1999
}

\section{SUMMARY}

In higher plants the gametophyte consists of a gamete in association with a small number of haploid cells, specialized for sexual reproduction. The female gametophyte or embryo sac, is contained within the ovule and develops from a single cell, the megaspore which is formed by meiosis of the megaspore mother cell. The dyad mutant of Arabidopsis, described herein, represents a novel class among female sterile mutants in plants. dyad ovules contain two large cells in place of an embryo sac. The two cells represent the products of a single division of the megaspore mother cell followed by an arrest in further development of the megaspore. We addressed the question of whether the division of the megaspore mother cell in the mutant was meiotic or mitotic by examining the expression of two markers that are normally expressed in the megaspore mother cell during meiosis. Our observations indicate that in dyad, the megaspore mother cell enters but fails to complete meiosis, arresting at the end of meiosis 1 in the majority of ovules. This was corroborated by a direct observation of chromosome segregation during division of the megaspore mother cell, showing that the division is a reductional and not an equational one. In a minority of dyad ovules, the megaspore mother cell does not divide. Pollen development and male fertility in the mutant is normal, as is the rest of the ovule that surrounds the female gametophyte. The embryo sac is also shown to have an influence on the nucellus in wild type. The dyad mutation therefore specifically affects a function that is required in the female germ cell precursor for meiosis. The identification and analysis of mutants specifically affecting female meiosis is an initial step in understanding the molecular mechanisms underlying early events in the pathway of female reproductive development.

Key words: Female gametophyte, Meiosis, Megasporogenesis, Megaspore mother cell, Arabidopsis thaliana

\section{INTRODUCTION}

The life cycle of plants alternates between a haploid gametophyte and a diploid sporophyte. In higher plants the gametophyte is reduced to a small number of cells surrounded by sporophytic tissue. The female gametophyte is contained within the ovule and consists of an embryo sac which harbors the egg cell and other associated haploid cells required for fertilization and development of the embryo sac and endosperm (Maheshwari, 1950; Willemse and van Went, 1984; Reiser and Fisher, 1993). The molecular mechanisms underlying female gametophyte development have begun to be elucidated with the identification of genes that act in the gametophyte and are necessary for its development (Drews et al., 1998; Grossniklaus and Schneitz, 1998). Mutations in genes that are required in the haploid phase of female gametophyte development would be expected to cause semisterility and show non-Mendelian segregation arising from reduced transmission through the affected sex. Female gametophytic mutants affecting development of the embryo sac have been identified in both
Arabidopsis and maize (Singleton and Manglesdorf, 1940; Nelson and Clary, 1952; Redei, 1965; Kermicle, 1971; Castle et al., 1993; Kieber et al., 1993; Niyogi et al., 1993; Springer et al., 1995; Feldman et al., 1997; Moore et al., 1997; Christensen et al., 1998). Another class of gametophytic mutants that affect embryo and endosperm development have also been identified (Ohad et al., 1996; Chaudhury et al., 1997; Grossniklaus et al, 1998). The recent cloning of gametophytic genes affecting female gametophyte development (Springer et al, 1995), as well as genes affecting embryo and endosperm development (Grossniklaus et al., 1998; Luo et al., 1998; Ohad et al., 1999) has provided valuable insights into the molecular events underlying this fascinating stage of the plant life cycle.

Less is known about genes that are involved in the control and elaboration of early steps in the female reproductive pathway, during megasporogenesis. The first identifiable step in the reproductive pathway at the anatomical level is the enlargement of a single cell in the subepidermal layer at the tip of the ovule primordium to form an archesporial cell. In Arabidopsis the archesporial cell directly forms the megaspore mother cell 
(mmc) and undergoes meiosis to give four haploid megaspores. Three of the spores undergo programmed cell death and the remaining one becomes the functional megaspore (Misra, 1962; Poliakova, 1964; Mansfield et al., 1991; Webb and Gunning, 1990). A large class of mutants that affect megasporogenesis has been identified in Arabidopsis, and shown to cause both male and female sterility (Schneitz et al., 1997). While a detailed analysis of these mutants has not been described, it is likely that many of these affect general meiosis, recombination and sporogenesis-related functions that would be common to both male and female reproductive development. The SPOROCYTELESS gene of Arabidopsis blocks formation of the sporocyte and encodes a transcriptional regulator that is localized to the nucleus (Yang et al., 1999). The SYN1 gene of Arabidopsis has been shown to be required for meiosis and encodes a $R A D 21$ homologue (Bai et al., 1999). A number of maize meiotic mutants have been described and shown to cause both male and female sterility (Curtis and Doyle, 1991). A particularly interesting mutant is the macl mutant of maize in which multiple archesporial cells are formed in a single ovule (Sheridan et al., 1996). These cells undergo megasporogenesis and megagametogenesis, resulting in a single ovule containing multiple embryo sacs at various stages of development. The macl mutant causes partial female sterility as well as complete male sterility. The male sterility is due to a block in prophase 1 of meiosis. In the afdl mutant of maize, the sporocyte undergoes an equational division instead of a reductional one, indicating that meiosis 1 is replaced by a mitotic division (Golubovskaya and Mashnenkov, 1975). In the ell mutant, meiosis 2 is affected leading to the production of unreduced spores (Rhoades, 1956).

Given the differences in form and function between male and female gametes, it would be expected that these two pathways of reproductive development are under the control of overlapping but distinct genetic programmes. The existence of female- and male-specific as well as non-specific gametophytic mutants acting at the haploid stage, after megasporogenesis, supports this notion. However, single gene mutations that specifically affect earlier stages of megasporogenesis without any effect in the male remain to be identified. We report here the isolation and characterization of $d y a d$, a novel femalespecific meiotic mutant of Arabidopsis.

\section{MATERIALS AND METHODS}

\section{Plant material and growth conditions}

Plants were grown in pots under fluorescent lights (7000 lux at $20 \mathrm{~cm}$ ) at $21^{\circ} \mathrm{C}$ according to conditions described by Somerville and Ogren (1982). EMS mutagenized $\mathrm{M}_{2}$ seed of Arabidopsis were purchased from Lehle and Co., Round Rock, Texas. Wild-type plants of ecotype Ler and No-O were used for backcrosses and mapping of the dyad mutant.

\section{Mutant Isolation and mapping}

EMS mutagenized $\mathrm{M}_{2}$ plants were grown and sterile plants were identified in which the siliques failed to elongate and contained no seed. Reciprocal crosses to wild type were done to determine if the cause of sterility was in the female, male, or both. In plants showing female sterility, ovules were examined after clearing (see below) to identify possible developmental defects. Mapping of dyad with SSLP markers was done using an $\mathrm{F}_{2}$ population derived from a cross of dyad in the Ler background to No-O. Dyad mutant plants were identified on the basis of sterility and embryo sac phenotype. DNA was isolated from
2-3 rosette leaves of $d y a d$ plants and used for PCR based mapping with microsatellite markers (Bell and Ecker, 1994). Primer pairs were purchased from Research Genetics Inc., Huntsville, Alabama.

\section{Light microscopy}

Flowers or inflorescences were fixed in FAA (3.7\% formalin, 5\% acetic acid, $50 \%$ ethanol) overnight at $4^{\circ} \mathrm{C}$, rinsed in $50 \%$ acetone, and dehydrated in an acetone series to $100 \%$ acetone. Tissue was cleared in methyl benzoate for 2 hours, followed by methyl benzoate:Spurr's resin (7:1) overnight. Ovules were dissected on a slide under a stereo dissecting microscope, mounted with a coverslip, and observed on a Zeiss Axioskop microscope under DIC optics using a $\times 40$ objective. Photography was done using low speed black and white film (15-25 ASA) which gives better details and contrast, and a $\times 40$ oil objective with a numerical aperture of 1.3 . Prints were scanned and edited using Adobe Photoshop 3.0.

For examination of developing microspores, buds were fixed and processed according to Altman et al. (1992). Anthers were dissected, squashed on a slide to release developing microspore tetrads and mounted with a coverslip.

\section{Fluorescence and confocal microscopy}

Pollen viability was measured using fluorescein diacetate according to the method of Heslop-Harrison and Heslop-Harrison (1970).

Aniline blue staining for callose detection in the mmc was carried out using dissected pistils after fixing in FAA and rinsing in water. Pistils were incubated in $0.1 \%$ aniline blue, $100 \mathrm{mM}$ Tris $\mathrm{pH} 8.5$, for $8-12$ hours. Ovules were dissected on a slide and mounted in 30\% glycerol with a coverslip. Fluorescence was observed on a Zeiss Axioskop microscope using a $365 \mathrm{~nm}$ excitation, $420 \mathrm{~nm}$ long-pass emission filter.

For confocal microscopy of meiotic chromosomes in the $\mathrm{mmc}$ pistils were dissected, Feulgen stained, and processed according to Braselton et al. (1996). Ovules were dissected on slides, mounted with a coverslip, and polymerized in LR-White (Electron Microscopy Sciences) resin at $60^{\circ} \mathrm{C}$. Observations of chromosomes were done on a Zeiss confocal microscope using a $\times 100$ oil objective. Excitation was at $488 \mathrm{~nm}$ and emissions were detected at $\geq 515 \mathrm{~nm}$.

\section{Electron microscopy}

For ultrastructural studies by transmission electron microscopy (TEM), pistils were fixed in $3 \%$ glutaraldehyde, $0.05 \mathrm{M}$ cacodylate $\mathrm{pH} 7$, for 2 hours, washed with water, followed by postfixation in $2 \% \mathrm{OsO}_{4}, 0.05$ $\mathrm{M}$ cacodylate, for 2 hours. Tissue was rinsed several times with distilled water and dehydrated in an acetone series. The $100 \%$ acetone step was repeated twice, followed by Spurr's resin: acetone (1:1), Spurr's: acetone (3:1) for 1 hour, and finally Spurr's for 24 hours. The resin was replaced and the tissue polymerized in an oven at $60-65^{\circ} \mathrm{C}$ for 2 days. Semithin $(0.5-1 \mu \mathrm{m})$ and thin $(0.1 \mu \mathrm{m})$ sections were cut on an ultramicrotome and examined by transmission electron microscopy.

\section{Analysis of AtDMC1 expression}

RNA in situ hybridization was carried out essentially according to the protocol of Jackson (1991) with modifications (Vielle Calzada et al., 1999). Digoxigenin-labelled antisense RNA was synthesized from an AtDMC1 cDNA clone (Klimyuk et al., 1997) using a Boehringer Mannheim kit (Cat. No. 1 175025) according to protocols supplied by the manufacturer and used as a probe. Sense RNA was used as a control probe.

For GUS-staining of ovules, pistils from plants carrying a $\mathrm{p} A t D M C 1-G U S$ fusion were dissected and placed in staining solution (Sundaresan et al., 1995) in a microtiter dish. The tissue was vacuum infiltrated and placed at $37^{\circ} \mathrm{C}$ for 1 day. Pistils were transferred to slides, ovules were dissected out, and mounted with a coverslip.

Observation was under DIC optics as described above and photographs were taken on Kodak Gold print film using a blue filter. Prints were scanned on a UMAX 3000 scanner and edited on Adobe photoshop 3.0. 


\section{RESULTS}

\section{The dyad mutation causes female sterility}

In order to identify mutants defective in female gametophyte development we screened approximately 22,000 EMS mutagenized $\mathrm{M}_{2}$ plants (derived from $20,000 \mathrm{M}_{1}$ plants) for female sterile mutants. We found 46 mutants with defects in ovule or female gametophyte development. Here we report on the characterization of one of the mutants, which showed a novel phenotype. The mutant was identified as a sterile plant with nonelongated siliques containing no seed. To determine the basis for sterility, we carried out reciprocal crosses to wildtype plants. The results clearly indicated the cause of sterility was in the female, and that pollen from mutant plants was fully fertile (Table 1). We also assayed for pollen viability in addition to checking for morphological abnormalities in the pollen, and failed to observe any differences from wild type. Pollen viability of the wild type was $88 \%$ (401/456) and for the mutant it was $86 \%(705 / 824)$. We examined cleared ovules to look for any morphological defect that would account for the observed female sterility. Pistils from open flowers and buds were fixed, cleared, dissected, and ovules examined by DIC microscopy. Mature ovules of the mutant lacked a differentiated embryo sac (Fig. 1B). Instead they contained two cells with prominent nuclei in the central region of the ovule where the embryo sac is normally located. The mutation therefore causes female-specific sterility.

\section{Genetic analysis}

The mutant was outcrossed as male to wild-type Arabidopsis ecotype Ler and No-O for genetic analysis and mapping. $\mathrm{F}_{1}$ plants were fertile, indicating that the mutation was recessive. In the first backcross generation we observed partial sterility in $F_{1}$ plants amounting to a 20-50\% reduction in seed set. However in subsequent backcrosses of the mutant to wild type we did not observe partial sterility in the $F_{1}$. The basis for the partial sterility observed initially in the $F_{1}$ was not pursued further. Segregation data for the dyad mutant phenotype in the $\mathrm{F}_{2}$ are shown in Table 2. Based on the data we cannot reject the hypothesis that the mutant phenotype segregates as a single gene recessive trait, but we can reject the hypothesis that the phenotype is due to two unlinked mutations. Mapping of the mutant using PCR-based markers indicated that it maps $3 \mathrm{cM}$ south of nga129 at the lower end of chromosome $5 \quad(90$ chromosomes examined). Linkage was also observed to a marker north, PHYC ( $\mathrm{R}=36 \%)$, to $\mathrm{g} 2368$ towards the south $(\mathrm{R}=20 \%)$, and not to markers on other chromosomes or other parts of chromosome 5 (data not shown).
Table 1. The dyad mutant causes female sterility

\begin{tabular}{lcc}
\hline Female parent & Male parent & No. of seeds per silique \\
\hline dyad & wild type & 0 \\
wild type & dyad & $29 \pm 13$ \\
wild type & wild type & $34 \pm 11$
\end{tabular}

Reciprocal crosses between dyad and wild type to measure seed yield were conducted and the results represent the mean and standard deviation from a minimum of 12 crosses.

Table 2. Segregation of the dyad phenotype

\begin{tabular}{lcc}
\hline \multicolumn{2}{c}{ Wild type: mutant } & \\
\hline Observed & Expected & $\chi^{2}$ \\
\hline $108: 29$ & $102.75: 34.25(3: 1)^{*}$ & $1.073, P>0.25$ \\
& $128.44: 8.56(15: 1) \ddagger$ & $52.06, P<<0.001$
\end{tabular}

*Mutant phenotype results from a single gene recessive mutation. † Two unlinked mutations are responsible for the mutant phenotype.

\section{Stages of ovule development in the dyad mutant}

To investigate the basis of the dyad phenotype and the origin of the two cells seen in ovules of the mutant, we carried out a stagewise analysis of development by examining cleared ovules using DIC microscopy. Early stages of reproductive development were as in wild type, with normal initiation of the ovule primordia and appearance of the mmc (Fig. 2E), and initiation of the inner and outer integuments (Fig. 2F). However, instead of undergoing a normal meiosis to give four spores, the mmc divides only once to give a dyad of two cells (Fig. 2G). Further divisions do not occur and the two cells persist through later stages of ovule development without degenerating (Fig. 2H) and are observed even in mature ovules at stages beyond when fertilization would normally have occurred (Fig. 1B). In a minority of dyad ovules (4\%) which we call class II, we observed that the mmc does not enter meiosis (Fig. 2I,J). Although this number is small, the
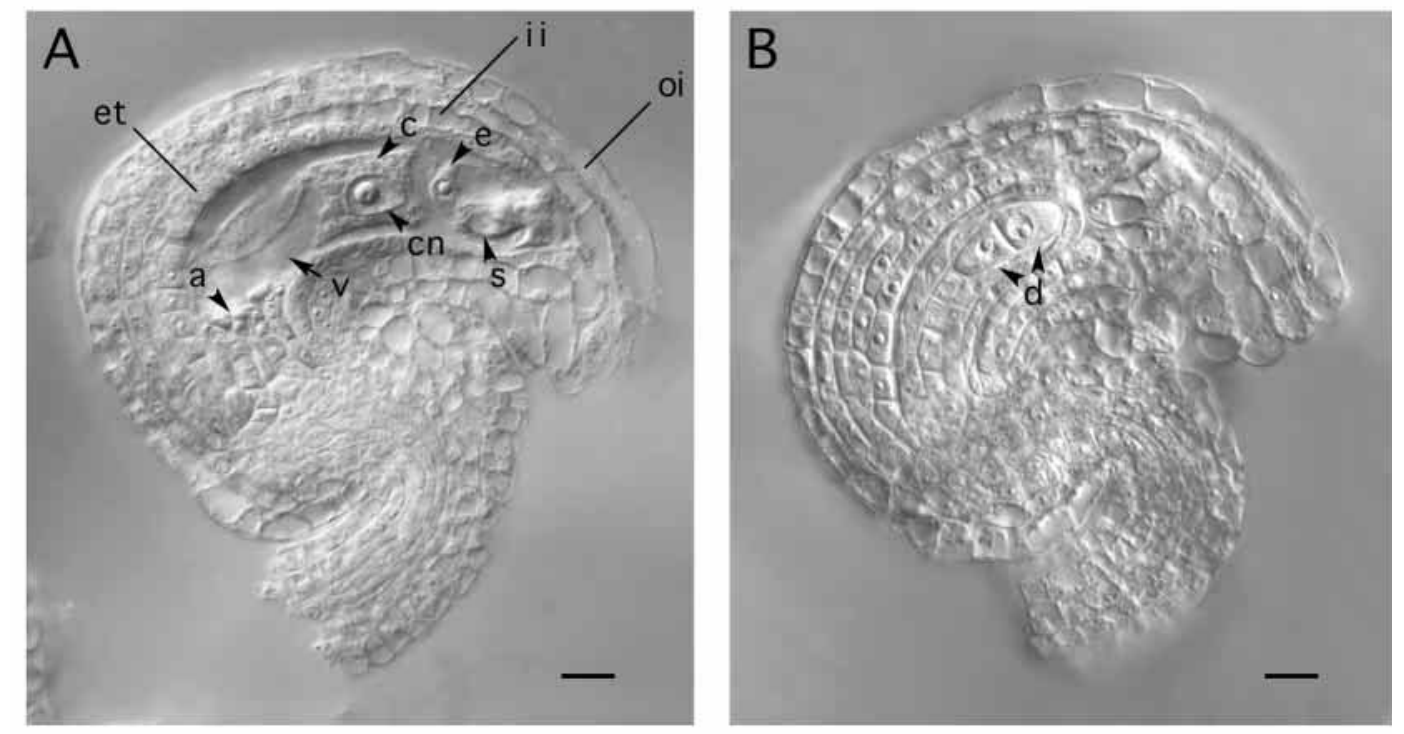

Fig. 1. Mature ovule of wild type (A) and dyad mutant (B). Optical sections of cleared ovules observed under DIC microscopy. a, antipodals; c, central cell; cn, central cell nucleus; e, egg cell; s, synergid; et, endothelium; ii, inner integument; oi, outer integument; d, dyad. Bar, $10 \mu \mathrm{m}$. 
observation is significant as it points to a low penetrance effect before or during meiosis 1 (see below and Discussion).

The development of the integuments and remainder of the sporophyte is normal and the ovule attains its eventual campylotropous (curved) configuration (Maheshwari, 1950) with both integuments extending beyond the nucellus and
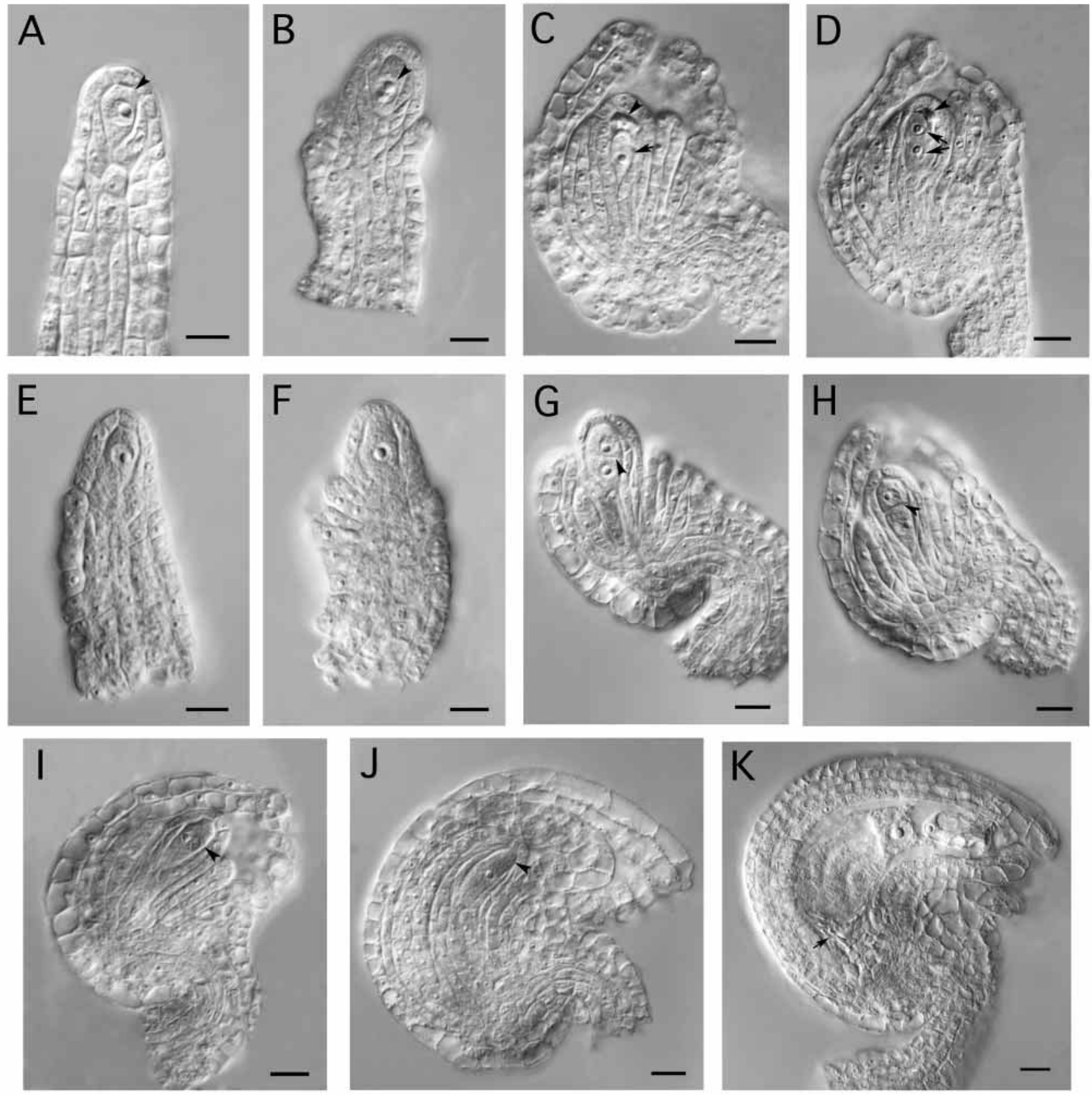

Fig. 2. Stages of female gametophyte development in wild type and dyad mutant. Ovule stages were determined from the development of the sporophyte using the nomenclature of Schneitz et al. (1995): 3-1, outer integument extends beyond nucellus; 3-2, outer integument surrounds nucellus; 3-4, inner integument extends beyond nucellar apex; endothelium differentiates; 3-6, inner integument forms an additional layer; 4, campylotropous ovule with micropyle next to funiculus. (A-D,K) Wild-type, (E-J)dyad. (A,E) Mmc has differentiated (arrowhead) and inner integument has initiated. (B,F) Premeiotic ovule showing integument primordia and enlarged mmc. Note that the nucleus (arrowhead) occupies a more proximal position towards the basal end of the cell in both dyad and wild type. $(\mathrm{C}, \mathrm{G})$ Postmeiotic ovule; in $d y a d$ the mmc has undergone a single division to give two cells separated by a cell wall (arrowhead), whereas in wild type, three of the four haploid spores formed by division of the mmc, degenerate (arrowhead). The arrow marks the functional megaspore. (D,H) Wild-type and dyad ovules at the 3-2 stage of ovule development. In dyad, megasporogenesis has arrested at the end of the first division of the mmc. Note the presence of degenerating spores (arrowhead) in D and two nuclei (arrows) without a cell wall. In $\mathrm{H}$ the two cells are separated by a cell wall (arrowhead). (I,J) Class II $d y a d$ ovules at the 3-2 and 3-6 stage containing an undivided mmc (arrowhead). Seven out of 172 ovules that ranged from the 3-2 stage to mature ovules contained a single $\mathrm{mmc}$ that had failed to divide. (K) Mature wild-type ovule with proximal nucellus degenerating (arrow). Bar, $20 \mu \mathrm{m}$ (A-J) and $15 \mu \mathrm{m}(\mathrm{K})$. 


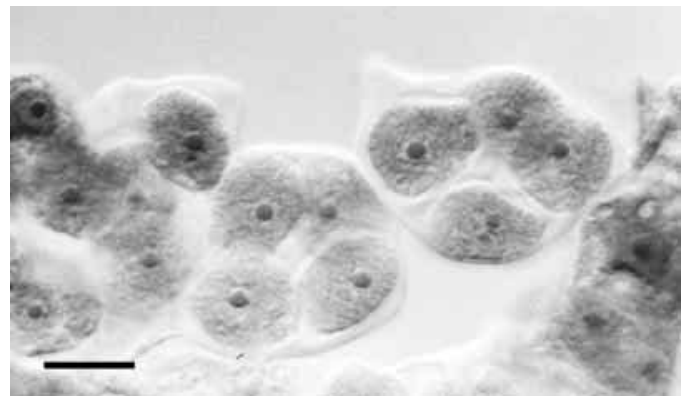

Fig. 3. Developing pollen grains of the dyad mutant showing normally developing microspore tetrads. Bar, $10 \mu \mathrm{m}$.

enclosing the micropyle. There is however, an interesting difference in the sporophyte, which is particularly striking in the case of older ovules. In wild-type ovules, the cells in the proximal nucellus below the embryo sac become irregular and degenerate (Fig. 2K). In the case of dyad this does not occur and four to five files of columnar cells can be observed in the proximal nucellus extending to the base of the dyad (Fig. 1B). We have also observed the lack of degeneration of the proximal nucellus in other mutants that lack an embryo sac (I. S., unpublished observations).
At the cellular level therefore, the basis for the dyad phenotype is that the mmc divides abnormally, giving a dyad instead of a normal tetrad. To determine if any of the pollen mother cells also showed this feature, we examined developing microspores for defects in meiosis and observed only normal tetrads with no apparent defects in male meiosis (Fig. 3).

\section{Megaspore mother cell division in the dyad mutant mmc polarity}

Initially, the mmc is unpolarized but becomes polarized shortly before meiosis with the nucleus occupying a more proximal location and a greater concentration of organelles (mitochondria and plastids) being found towards the chalazal end (Willemse, 1981; Willemse and van Went, 1984). It is possible that the aberrant division of the $\mathrm{mmc}$ and subsequent arrest seen in the dyad mutant is the consequence of loss of cell polarity and this might be evidenced by an altered distribution of intracellular organelles. As a test of this possibility we carried out an ultrastructural examination of the mmc by transmission electron microscopy.

The mmc at early stages is unpolarized with respect to distribution of organelles (data not shown) but prior to meiosis it becomes elongated and polarized (Fig. 4A). We observed that the mmc in dyad also shows polarity with a concentration of
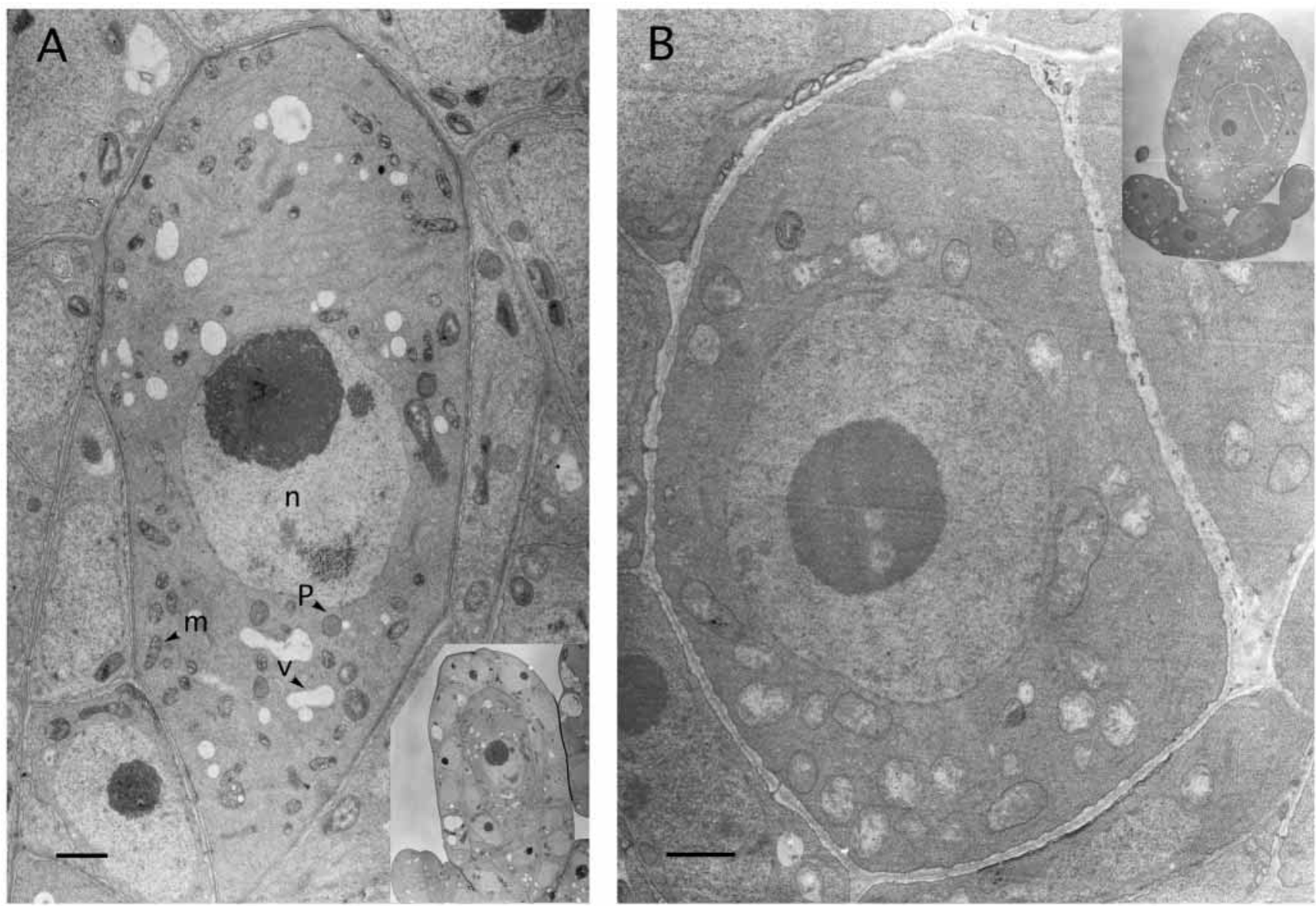

Fig. 4. Mmc polarity in dyad. Mmc ultrastructure in wild type (A) and dyad (B) seen by transmission electron microscopy. Insets at lower magnification show the nucellar region containing the mmc and early integuments. (A) Longitudinal section of a mmc shortly before meiosis showing a concentration of organelles at the chalazal end ( $m$, mitochondria; $n$, nucleus; $p$, plastids; $v$, vacuole). The upper region of the cell above the nucleus is relatively depleted of organelles. (B) Longitudinal/oblique section showing concentration of organelles towards the chalazal end of the mmc in dyad. Bar, $2.5 \mu \mathrm{m}$. 
organelles at the chalazal end (Fig. 4B). Therefore there does not appear to be a loss in polarity of the mmc at least at the ultrastructural level and the cause of the aberrant division may lie elsewhere.

\section{The mmc in dyad undergoes a defective meiosis}

A fundamental issue in understanding the effect of the dyad mutation is the nature of the division of the mmc in the mutant and whether it is meiotic or mitotic. We addressed this question using three experimental approaches. First we examined the dividing mmc for the presence of callose, a crosslinked polysaccharide that is synthesized in the mmc during meiosis prior to cytokinesis. The presence of callose can be readily detected by aniline blue fluorescence, which therefore acts as a convenient cytological marker for meiosis in the mmc (Rodkiewicz, 1970). In wild type, fluorescence first appears in the mmc during meiosis shortly before cytokinesis and becomes concentrated at the site of the newly forming cell plate (Fig. 6C). Subsequently the fluorescence gets concentrated towards the micropylar end and ultimately in the degenerating spores (Schneitz et al., 1995; data not shown). By the time developing embryo sacs reach the $4 \mathrm{~N}$ stage, the material from the degenerating megaspores has been removed and aniline blue fluorescence is no longer visible (data not shown). In $d y a d$, aniline blue fluorescence is observed in the dividing $\mathrm{mmc}$ and is strongest at the site of the cell plate
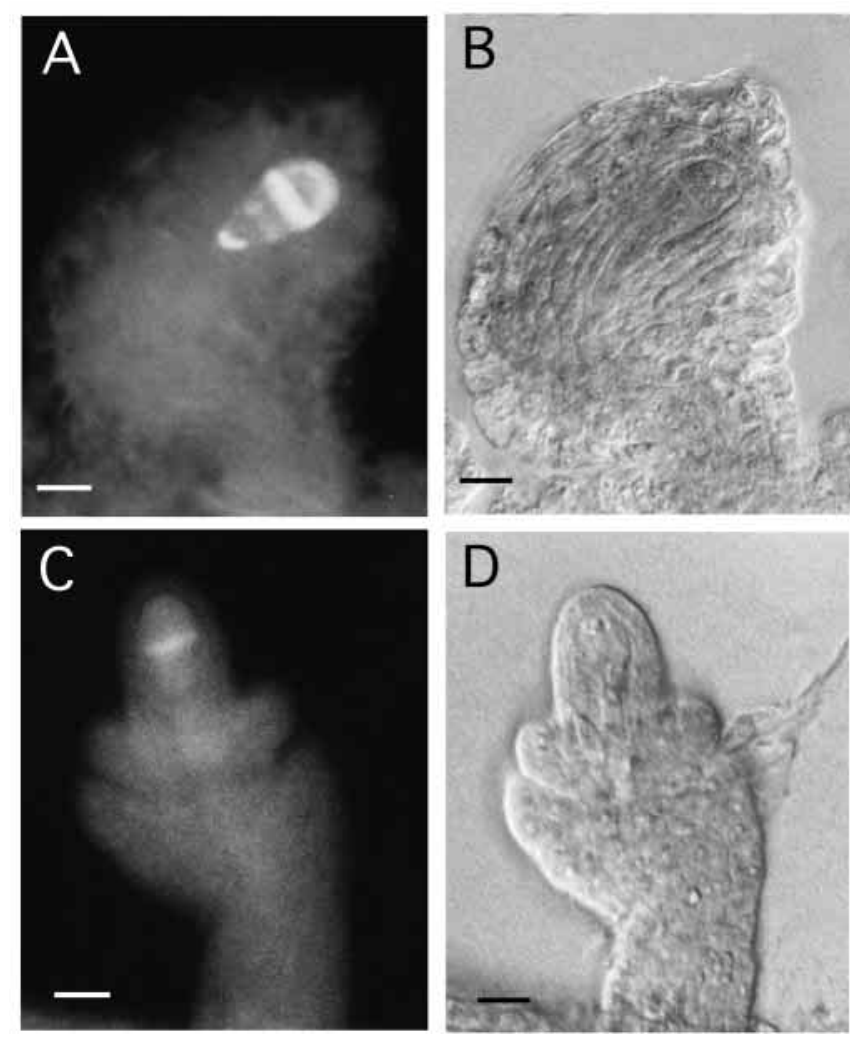

Fig. 5. Callose production in the mmc. (A,B) dyad, (C,D) wild type. $(\mathrm{A}, \mathrm{C})$ Aniline blue fluorescence image. (B,D) The corresponding ovules viewed under DIC optics. Fluorescence is seen in both dyad and wild type and is highest at the cell plate in the dividing mmc. These ovules were not cleared, hence the DIC image does not reveal internal details of the mmc. Bar, $10 \mu \mathrm{m}$.
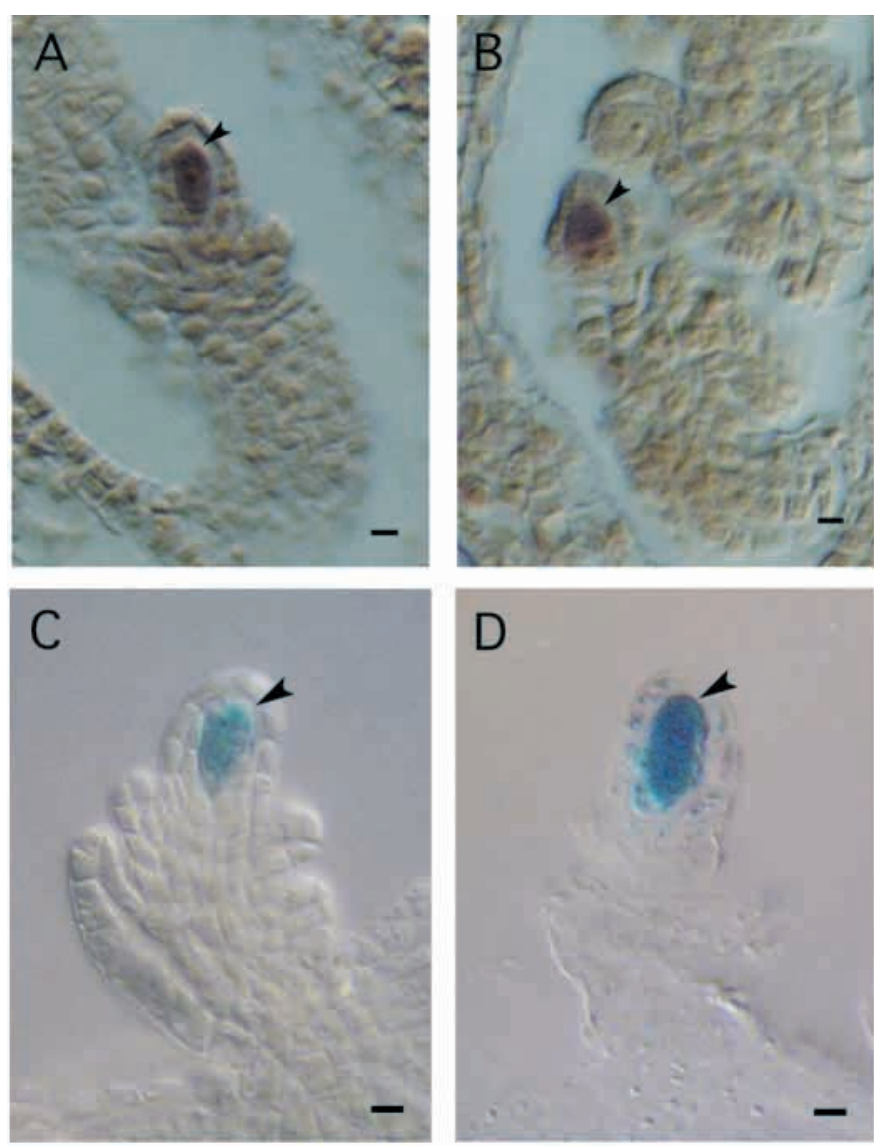

Fig. 6. AtDMCl expression in the mmc. (A,B) RNA in situ hybridization of AtDMCI antisense RNA to ovule sections. Specific hybridization is seen in the mmc (arrowhead) at meiosis in (A) dyad and (B) wild type. Controls using a sense RNA probe gave no signal. (C,D) pAtDMCl-GUS expression in the mmc at meiosis (arrowhead) in dyad (C) and wild type (D). Bar, $5 \mu \mathrm{m}$.

separating the two cells of the dyad (Fig. 5A). In some cases we have observed fluorescence in dyad ovules even at later stages of development beyond when it would normally be found in the wild type (data not shown). This would imply that callose persists and is not removed in these ovules. Overall, the frequency of mmcs showing aniline blue fluorescence in the dyad mutant (33/41 ovules) is comparable though slightly lower than that for wild type (37/37 ovules). These data would suggest that in $d y a d$, the division of the mmc is a meiotic one.

\section{AtDMC1 expression}

As discussed above, female meiosis is associated with the appearance of callose, which can therefore be considered a cytological marker for meiosis. However, this involves making certain assumptions. The exact relationship between meiosis and callose production is unknown and it is possible that the two are under the control of contemporaneous but parallel and distinct developmental pathways. To address this issue, we examined the expression of a molecular marker AtDMC1, which has been shown to be specifically expressed during meiosis in both the male and female lineage in Arabidopsis (Klimyuk and Jones, 1997). We examined expression of AtDMCl in the mmc using RNA in situ hybridization to 

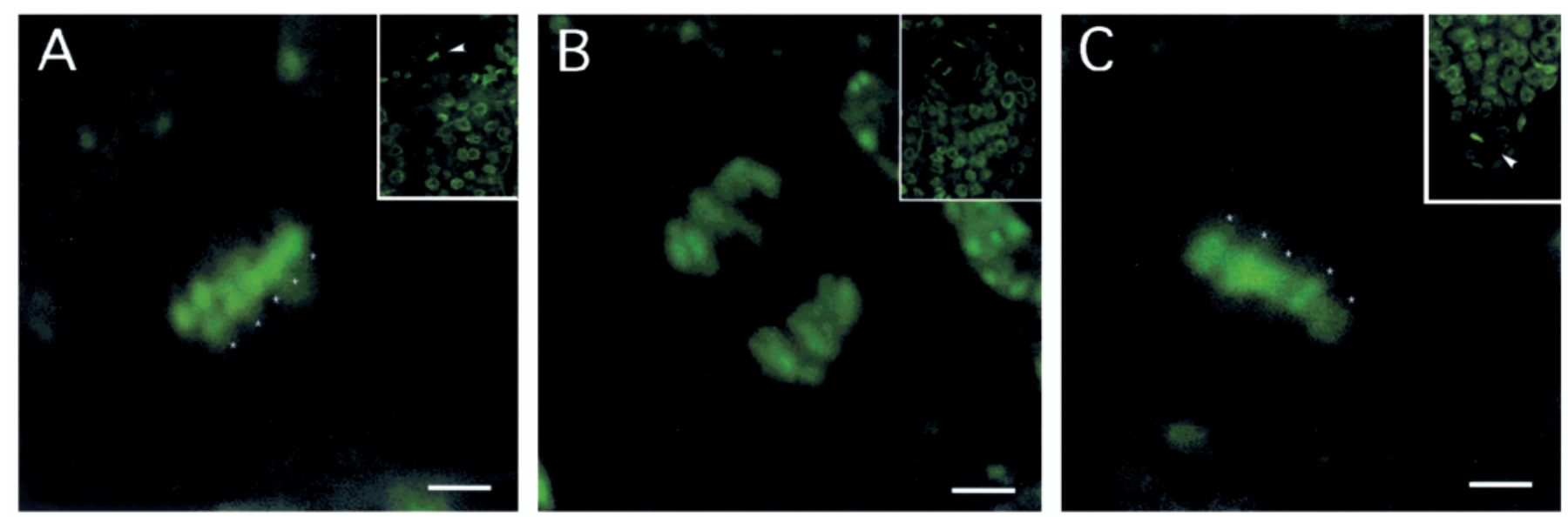

Fig. 7. Chromosome segregation in the dividing mmc. Confocal microscopy of chromosomes in the mmc, equatorial views. (A,B) dyad, and (C) wild type. (A) Early anaphase showing two sets of five chromosomes (*) starting to move apart. (B) Mid anaphase: each set contains five chromosomes. (C) Late metaphase showing five chromosome pairs $(*)$. Insets show ovules with mmc at low magnification. Bar, $2 \mu$ m.

sections of mutant and wild-type inflorescences. The results clearly indicate that $A t D M C l$ is expressed in the mmc in dyad ovules at the stage when meiosis occurs (Fig. 6A).

We also examined expression of a GUS reporter gene driven by the AtDMCl promoter in plants carrying the dyad mutation. GUS expression is detected in the mmc at the time of meiosis in both wild type and the dyad mutant (Fig. 6C,D).

\section{Confocal microscopy of meiotic chromosomes}

As a final test, we sought to directly examine chromosome segregation during division of the mmc. Arabidopsis chromosomes are small in size and each ovule contains a single mmc, enclosed within the integuments. Hence observations on female meiosis are more difficult when compared to male meiosis, using conventional cytogenetic methods. To visualize chromosomes, we carried out Feulgen staining of pistils, followed by dissection and embedding of ovules in resin. Ovules were then examined using confocal laser scanning microscopy (Braselton et al., 1996). We were able to identify ovules in which the mmc was undergoing division and chromosomes were at metaphase or early anaphase of meiosis 1. Both mutant (Fig. 7A) and wild-type (Fig. 7C) metaphase plates were observed and each half-plate was seen to consist of five chromosomes. Hence the division in dyad is a reductional and not an equational one.

Taken together these data indicate that the mmc in dyad enters meiosis and completes meiosis 1 but is blocked at the stage of entry into meiosis II.

\section{Variation in mmc division in dyad}

Ovules carrying two cells in the position normally occupied by the embryo sac make up the largest single class in post stage 3-1 ovules of the dyad mutant (Table 3). This observation is consistent with the primary defect being early in meiosis and most ovules not progressing beyond the first division. However, as noted above we also see a significant number (26/581, or $4 \%$ of post stage 3-1 ovules), in which the mmc does not divide. Therefore dyad may play a role in entry into or during meiosis 1 and the undivided mmc may represent the higher expressivity phenotype. We did not observe undivided mmcs in wild-type ovules at stage 3-1 or later $(0 / 357)$, and whenever the developing gametophyte in these ovules consisted of a single uninucleate cell, it was always accompanied by material from the degenerating megaspores. The undivided mmc could be unambiguously distinguished from a functional megaspore in wild type, and undivided mmcs were never observed in wild-type ovules at stage 3-1 or later. By contrast, in the dyad mutant, we saw only undivided mmcs, and no functional megaspores accompanied by degenerating sister spores (except in 3/581 ovules). We therefore infer that the undivided cells observed are mmcs, and not arrested megaspores with all traces of the sister spores having been removed. In cases where more divisions do occur in dyad, the upper limit of the number of cells formed is four. The cells that are formed by division of the mmc in $d y a d$, therefore, probably represent the four spores, albeit defective ones that are unable to form a functional gametophyte.

The plane of division of the mmc in dyad is most commonly transverse although oblique and longitudinal divisions are also seen (Fig. 8A,B). Several ovules show additional divisions of one or both of the dyad cells (Fig. 8C-E; Table 3). Multinucleated cells are also seen in many ovules. The pattern and number of divisions is variable and we have observed up to seven nuclei in one cell (Fig. 8F). In some cases we also observe structures reminiscent of a developing embryo sac at the $2 \mathrm{~N}$ stage (Fig. 8G). This raises the question of whether some parts of the program of embryo sac development following megasporogenesis may be turned on even though meiosis has been defective and incomplete. Although additional divisions of one or both of the dyad nuclei are frequently seen we have never observed a normally developing or differentiated embryo sac and indeed, the $d y a d$ mutant fails to set any seed. Taken together, our observations demonstrate that the dyad mutation specifically affects a function that is required in the female germ cell precursor during meiosis and megasporogenesis.

\section{DISCUSSION}

In all sexually reproducing organisms, the gametes develop from haploid cells formed after meiosis. Meiosis is therefore a 
Fig. 8. Variation in mmc division in dyad. The region of ovules containing meiotic products or the developing female gametophyte are shown. Ovules were at different developmental stages. (A-E) Stage 2-5 to 3-4 ovules. (F-K) Late stage mature ovules corresponding to postfertilization stages for wild type. (A) Oblique division. (B) Longitudinal division. (C) Linear triad. (D) T-shaped tetrad. (E) Linear tetrad. The uppermost cell has undergone an additional nuclear division (arrowhead). (F) A seven-nucleate cell. Note the absence of a vacuole that would be present after stage 3-4 in a wild-type embryo sac. (G) A twonucleate cell with a vacuole resembling a stage 3-3 embryo sac. Arrowheads show nuclei. The lower cell of the dyad has degenerated and residual material is visible. $(\mathrm{H})$ The lower cell of the dyad has become vacuolated. (I) Each cell of the dyad contains 3 nuclei (arrowheads).

(J) Two cells each containing 2 nuclei. (K) The upper cell has undergone an additional nuclear division while the lower one has remained uninucleate. Bar, $7.5 \mu \mathrm{m}$.
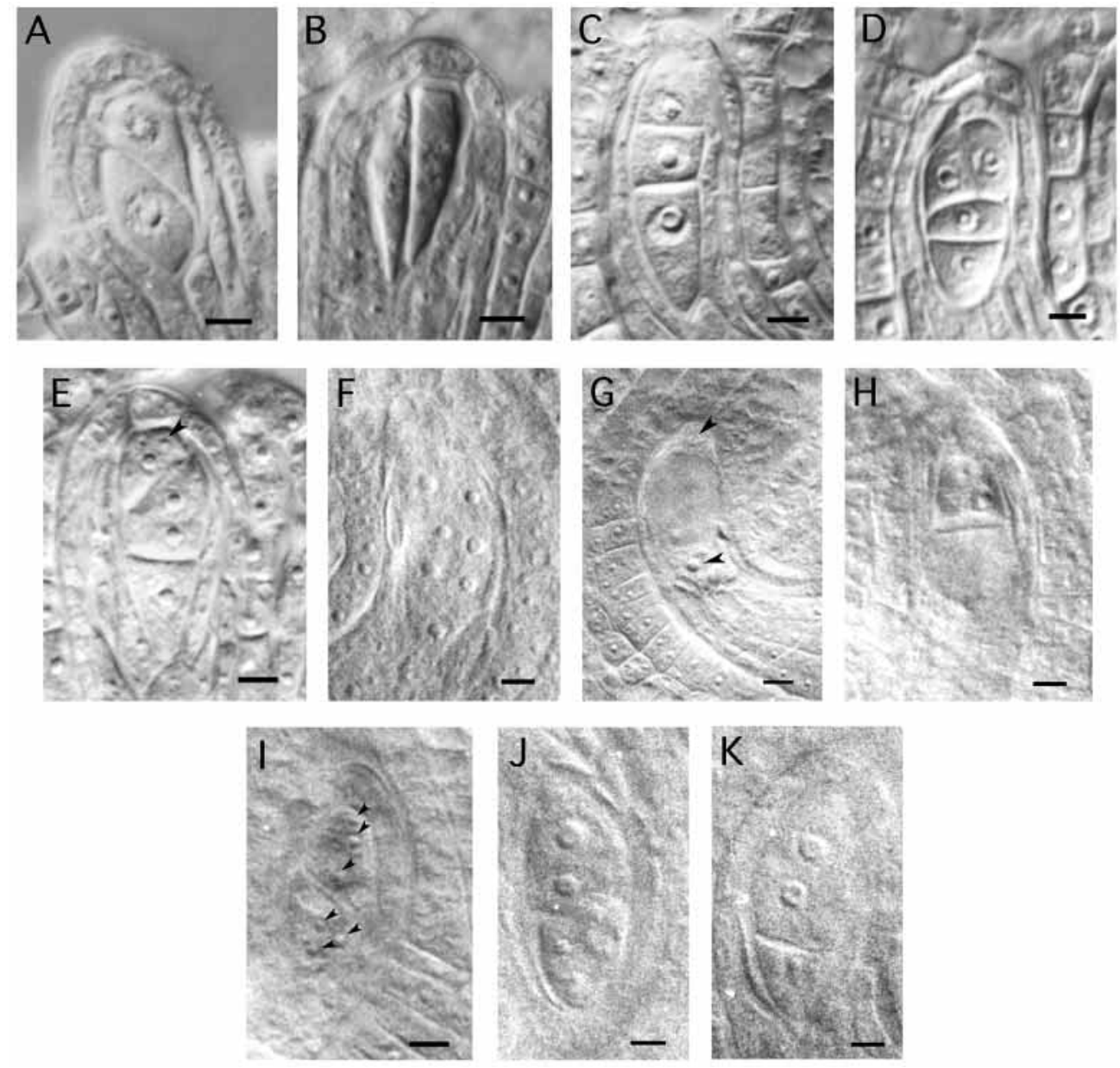

key step leading into the pathway of gametogenesis. In higher plants there is sexual dimorphism between the megaspore and microspore mother cells that are the precursors to the female and male spores respectively. Most mutations affecting meiosis affect both sexes, implying that a common set of genes required for meiosis are integrated into two overlapping but distinct developmental pathways leading to the formation of the male and female spores. The properties of the dyad mutant of Arabidopsis described above indicate that it specifically affects a function required for female meiosis without any effect in the male. It is however, possible that the mutation could be a female-specific allele of a gene that also acts elsewhere.

The dyad mutant was identified in a screen for female sterile mutants of Arabidopsis among a population of EMS mutagenized $\mathrm{M}_{2}$ plants. An examination of cleared mature ovules showed that in place of an embryo sac, two large cells were present. Pollen development, however, was normal, and both morphological examination and pollen viability tests, as well as outcrosses of the mutant as male to wild type as female showed no evidence of any abnormality in the pollen. The mutant is thus female sterile but male fertile. The two cells

Table 3. Megaspore mother cell division products in dyad

\begin{tabular}{|c|c|c|c|c|c|c|c|}
\hline \multirow{2}{*}{$\begin{array}{l}\text { Ovule } \\
\text { stage }\end{array}$} & \multicolumn{5}{|c|}{ Number of ovules } & \multirow{2}{*}{$\begin{array}{l}\text { Multinuc. } \\
\text { cells }\end{array}$} & \multirow{2}{*}{$\begin{array}{l}\text { Number } \\
\text { of ovules }\end{array}$} \\
\hline & 1 cell & 2 cell & 3 cell & 4 cell & $>4$ cells & & \\
\hline $3-1$ & 15 & 82 & 0 & 0 & 0 & 2 & 97 \\
\hline $3-2$ & 8 & 88 & 13 & 3 & 0 & 11 & 112 \\
\hline $3-4$ & 6 & 82 & 33 & 19 & 0 & 17 & 140 \\
\hline $3-6$ & 6 & 74 & 58 & 36 & 0 & 38 & 174 \\
\hline 4 & 6 & 74 & 46 & 25 & 2 & 15 & 155 \\
\hline Total & 41 & 400 & 150 & 83 & 2 & 83 & 678 \\
\hline
\end{tabular}

Division products of the mmc at different stages of ovule development in dyad. Ovules were scored on the basis of ovule stage (see Fig. 2 legend for assignment of ovule stages) and the number of cells formed by division of the mmc. A total of 680 ovules were scored, over a range of stages. In 2 cases we observed 5 cells, and in another 2 cases in stage 4 ovules we saw a 4-nucleate and a 6-nucleate structure which resembled a developing embryo sac with a vacuole. 
observed in the majority of dyad ovules, are the products of a single division of the mmc followed by an arrest of subsequent divisions. Unlike the case in wild type where three of the four spores formed after meiosis undergo programmed cell death, both the cells seen in dyad persist through later stages of ovule development and are present in mature ovules. This suggests the presence of a developmental checkpoint ensuring that programmed cell death of the megaspores is only initiated after completion of meiosis. The sporophytic parts of the ovule develop normally.

We did notice a difference in the sporophyte between $d y a d$ and wild type. In wild-type ovules, growth and expansion of the embryo sac is accompanied by degeneration of cells in the proximal nucellar region below the embryo sac. By contrast in dyad, 4-5 rows of cells are retained in the proximal nucellus through the later stages of ovule maturation. The persistence of nucellar cells may be an indirect effect of the mutation arising from the absence of an embryo sac as we have observed the same in other mutants that lack an embryo sac. It is therefore likely that the degeneration of the proximal nucellar cells is mediated directly or indirectly by the embryo sac. Thus, in addition to the development of the gametophyte being closely dependent upon and influenced by the surrounding maternal tissue (Ray, 1998), our evidence shows that the gametophyte also influences the sporophyte.

The mmc is a polarized structure in which organelles (mitochondria and plastids) become concentrated at the chalazal end of the cell towards later stages of its development, just before the start of meiosis. This polarization probably reflects the fact that it is the chalazal spore that becomes functional, whereas the other three degenerate. The role of asymmetric cell division and the unequal partitioning of cytoplasmic factors as a means of directing daughter cells towards different developmental fates is well documented in a number of animal as well as plant systems (Horvitz and Herskowitz, 1992; Jan and Jan, 1998; Twell et al., 1998). In the case of megasporogenesis, several studies have implicated cellular polarity as being important in the specification and development of the functional megaspore (Willemse and van Went, 1984; Webb and Gunning, 1990; Huang and Russel, 1993). We considered the possibility that the defect in meiosis and megasporogenesis in dyad is due to a defect in polarity of the mmc and partitioning of cytoplasmic factors. Such a loss of polarity could lead to a visible alteration in the distribution of organelles, towards the chalazal end of the cell. An examination of the mmc by transmission electron microscopy failed to reveal any differences between the mutant and wild type, suggesting that polarity is still present in the mmc at least at the gross level. It does not of course rule out more subtle molecular defects in polarity that would not have been obvious at the TEM level.

Two possibilities arise when considering the basis of the dyad phenotype. One is that the mmc does not enter meiosis but instead undergoes a mitotic division to give rise to two cells that then arrest. Twin mmcs have been reported to occur in wild-type Arabidopsis (Schneitz et al., 1995) at a low frequency (in about $3 \%$ of ovules) and this could bear some resemblance to what happens in dyad. The existence of a mechanism by which a mmc once formed would inhibit adjacent cells from adopting a sporogenic pathway of development is one way of restricting the number of mmcs to one per ovule. In most plant species, there is one mmc per ovule although in some families such as the Amentiferae, Casuarinaceae, Compositae, Rosaceae and Ranunculaceae, more than one cell is found in some representatives (Maheshwari, 1950). The macl mutant of maize (Sheridan et al., 1996) forms multiple archesporial cells in the ovule and these undergo meiosis and embryo sac development to different extents. In addition to its effect in the female, the macl mutant is also defective in pollen meiosis at prophase 1 . An alternative possibility in the case of dyad is that the mmc enters meiosis and completes the first division but does not carry out meiosis II. Several lines of evidence support this and taken together, rule out the possibility that the mmc undergoes a mitotic division to give rise to two sporogenous cells, each of which inhibits the further development of the other.

Firstly, callose, a cytological marker that appears in the mmc during meiosis is observed in both mutant and wild type. Secondly, AtDMC1, a molecular marker that is specifically expressed in the megaspore and microspore mother cells at the time of meiosis, is also expressed in the mmc in dyad. Finally, examination of segregating chromosomes during metaphase and anaphase of the dividing mmc using confocal laser scanning microscopy shows that it undergoes a reductional division. The mmc in dyad enters meiosis, completes meiosis 1 , and arrests at the end of the first division in the majority of ovules.

In Arabidopsis, it has been observed that cellularization of the four spores occurs after both nuclear divisions have taken place in meiosis (Webb and Gunning, 1990; Schneitz et al., 1995). In $d y a d$, the two cells formed are clearly separated by a cell wall implying that it is the rapidity with which the second nuclear division follows the first that is responsible for cellularization occurring after it is complete. The second division is not essential for cellularization and when it does not occur, cellularization still takes place.

While the predominant mutant phenotype is the presence of a dyad, there is considerable variation in the number of divisions of the mmc. Based on the data in Table 3, by stage $3-1$, the mmc in about $85 \%$ of ovules has undergone meiosis 1 whereas in $15 \%$ it has not. Of the $15 \%$ of mmcs that have not divided, about two-thirds undergo a delayed meiosis whereas one-third do not divide and can be observed even in late stage ovules. The number of mmc divisions is variable with the upper limit being four cells, consistent with the view that the products of mmc division are defective megaspores. The effect of the dyad mutation therefore extends from the beginning of meiosis, with a small proportion of mmcs $(4 \%)$ not undergoing meiosis 1. About half the mmcs progress through meiosis 1 but do not undergo meiosis 2 , and the remainder form a triad or a tetrad which does not develop further in the majority of cases. In addition we observed multinucleate cells which have also been seen in other meiotic mutants. In some cases we observed structures that resemble a developing gametophyte at the $2 \mathrm{~N}$ stage in one of the cells of the dyad. This raises the possibility that in Arabidopsis, parts of the program underlying development of the female gametophyte can sometimes become uncoupled from the completion of meiosis. In diplosporous apomictic species, meiosis 1 is aberrant or bypassed and the mmc gives rise to two unreduced spores one of which forms the embryo sac, while the other degenerates. Whether any of the cells of the dyad express genes that are 
normally turned on during later stages of embryo sac development remains to be seen and we are currently examining the expression of molecular markers that are expressed in parts of the female gametophyte at different developmental stages.

The DYAD gene may encode a positive regulator required for entry into meiosis 2 . Genes that affect the second meiotic division in other species are known to affect the cell cycle, and in some cases have been shown to interact with components that are central to cell cycle control (O'Keefe et al., 1989; McCarroll and Esposito, 1994; Gonczy et al., 1994). It is possible that the dyad gene may act in a related manner. Alternatively, the arrest in dyad could be the consequence of a defect in meiosis 1 , and a failure to pass a checkpoint control at the end of the first division. Although the predominant phenotypic class is an ovule containing a dyad, a small but significant number of ovules $(4 \%)$ contained a mmc that had not undergone the first division and this would point to a role for the dyad gene prior to or early in meiosis 1 . Cloning and molecular characterization of the dyad gene should provide information to distinguish between these possibilities and also shed light on processes that are unique to the female lineage during meiosis and megasporogenesis in flowering plants.

We would like to thank Dr Jean-Philippe Vielle-Calzada for valuable discussions, and advice and help with in situ hybridization and microscopy. We are grateful to Dr Victor Klimyuk and Dr Jonathan Jones for providing the AtDMC1 cDNA clone and seeds of pAtDMC1-GUS transformed lines. We would like to thank Tamara Howard for expert assistance with electron microscopy, Bhavna Agashe for help with mapping, Dr David Jackson for advice on in situ hybridization, and Dr J. Dhawan for critical comments on the manuscript. This work was funded by the Council for Scientific and Industrial Research, a grant from the Department of Biotechnology, India (DBT) to Imran Siddiqi, and in part, by a competitive grant award from Pioneer Hi-bred International to Ueli Grossniklauss. Gopal Ganesh was supported by a DBT postdoctoral fellowship. Travel support from the Dorabji Tata Trust to I. S. during the course of this work is also acknowledged as is supply of seed material and plasmids from the ABRC. Imran Siddiqi especially thanks Prof. James Watson for his interest and support through the Oliver Grace Endowment, during a Cold Spring Harbor Visiting Fellowship.

\section{REFERENCES}

Altman, T., Damm, B., Halfter, U., Willmitzer, L. and Morris P.-C. (1992). Protoplast transformation and methods to create specific mutants in Arabidopsis thaliana. In Methods in Arabidopsis Research. (ed. Koncz, C., Chua, N.-H. and Schell, J.). Singapore: World Scientific Publishing Co.

Bai, X., Peirson, B. N., Dong, F., Cai, X. and Makaroff, C. A. (1999). Isolation and characterization of SYN1, a RAD21-like gene essential for meiosis in Arabidopsis. Plant Cell 11, 417-430.

Bell, C. J. and Ecker, J. R. (1994). Assignment of 30 microsatellite loci to the linkage map of Arabidopsis. Genomics 19, 137-144.

Braselton, J. P., Wilkinson, M. J. and Clulow, S. A. (1996). Feulgen staining of intact plant tissue for confocal microscopy. Biotech. Histochem. 71, 8487.

Castle, L. A., Errampalli, D., Atherton, T. L., Franzmann, L. H., Yoon, E. S. and Meinke, D. W. (1993). Genetic and molecular characterization of embryonic mutants identified following seed seed transformation in Arabidopsis. Mol. Gen. Genet. 241, 504-514.

Chaudhury, A. M., Ming, L., Miller, C., Craig, S., Dennis, E. S. and Peacock, W. J. (1997). Fertilization-independent seed development in Arabidopsis thaliana. Proc. Natl. Acad. Sci. USA 94, 4223-4228.

Christensen, C. A., Subramanian S. and Drews, G. N. (1998). Identification of gametophytic mutations affecting female gametophyte development in Arabidopsis. Dev. Biol. 202, 136-151.

Curtis, C. A. and Doyle, G. G. (1991). Double meiotic mutants of maize: implications for the genetic regulation of meiosis. J. Hered. 82, 156-163.

Drews, G. N., Lee, D. and Christensen, C. A. (1998). Genetic control of female gametophyte development and function. Plant Cell 10, 1-15.

Feldman, K. A., Coury, D. A. and Christianson, M. L. (1997). Exceptional segregation of a selectable marker (KanR) in Arabidopsis identifies genes important for gametophytic growth and development. Genetics 147, 14111422.

Golubovskaya, I. N. and Mashnenkov, A. S. (1975). Genetic control of meiosis 1. Meiotic mutation in corn (Zea mays L.) afd, causing the elimination of the first meiotic division. Genetika 11, 11-17.

Gonczy, P., Thomas, B. J. and DiNardo, S. (1994) ROUGHEX is a dosedependent regulator of the second meiotic division during Drosophila spermatogenesis. Cell 77, 1015-1025.

Grossniklaus, U. and Schneitz, K. (1998). The molecular genetic basis of ovule and megagametophyte development. Semin. Cell Dev. Biol. 9, 227238.

Grossniklaus, U., Calzada, J-P. V., Hoeppner, M. A. and Gagliano, W. B. (1998). Maternal control of embryogenesis by MEDEA, a Polycomb group gene in Arabidopsis. Science 280, 446-450.

Heslop-Harrison, J. and Heslop-Harrison, Y. (1970). Evaluation of pollen viability by enzymatically induced fluorescence; intracellular hydrolysis of fluorescein diacetate. Stain Technol. 45, 115-120.

Horvitz, R. H. and Herskowitz, I. (1992). Mechanisms of asymmetric cell division: two Bs or not two Bs, that is the question. Cell 68, 237-255.

Huang, B.-Q. and Russel, S. D. (1993). Polarity of nuclear and plastid DNA in megasporogenesis and megagametogenesis of Plumbago zeylanica. Sex. Plant Reprod. 6, 205-211.

Jackson, D. P. (1991). In situ hybridization in plants. In Molecular Plant Pathology: A Practical Approach (ed. Bowles, D. J., Gurr, S. J. and McPherson, M.) Oxford University Press.

Jan, Y. N. and Jan, L. Y. (1998). Asymmetric cell division. Nature 392, 775778.

Kermicle, J. L. (1971). Pleiotropic effects on seed development of the indeterminate gametophyte gene of maize. Am. J. Bot. 58, 1-7.

Kieber, J. J., Rothenberg, M., Roman, G., Feldman, K. A. and Ecker, J. R. (1993). CTR1, a negative regulator of the ethylene response pathway in Arabidopsis, encodes a member of the Raf family of protein kinases. Cell 72, 427-441.

Klimyuk, V. I. and Jones, J. D. G. (1997) AtDMC1, the Arabidopsis homologue of the yeast $D M C 1$ gene: characterization, transposon-induced allelic variation and meiosis-associated expression. Plant J. 11, 1-14.

Luo, M., Bilodeau, P., Koltunow, A., Dennis, E. S., Peacock, W. J. and Chaudhury, A. M. (1999). Genes controlling fertilization-independent seed development in Arabidopsis thaliana. Proc. Natl. Acad. Sci. USA 96, 296301 .

Maheshwari, P. (1950). An Introduction to the Embryology of Angiosperms. New York: McGraw-Hill.

Mansfield, S. G., Briarty, L. G. and Erni, S. (1991). Early embryogenesis in Arabidopsis thaliana. I. The mature embryo sac. Can. J. Bot. 69, 447460.

McCarroll R. M. and Esposito R. E. (1994) SPO13 negatively regulates the progression of mitotic and meiotic nuclear division in Saccharomyces cerevisiae Genetics 138, 47-60.

Misra, R. C. (1962). Contribution to the embryology of Arabidopsis thaliana (Gay and Monn.). Agra Univ. J. Res. Sci. 11, 191-199.

Moore, J. M., Calzada, J.-P. V., Gagliano, W. and Grossniklauss, U. (1997). Genetic characterization of hadad, a mutant disrupting female gametogenesis in Arabidopsis thaliana. Cold Spring Harbor Symp. Quant. Biol. 62, 35-47.

Nelson, O. E. and Clary, G. B. (1952). Genetic control of semisterility in maize. J. Hered. 43, 205-210.

Niyogi, K. K., Last, R. L., Fink, G. R. and Keith, B. (1993). Suppressors of trp1 fluorescence identify a new Arabidopsis gene, TRP4, encoding the anthranilate synthase beta subunit. Plant Cell 5, 1011-1027.

Ohad, N., Margossian, L., Hsu, Y.-C., Williams, C., Repetti, P. and Fischer, R. L. (1996). A mutation that allows endosperm development without fertilization. Proc. Natl. Acad. Sci. USA 93, 5319-5324.

Ohad, N., Yadegari, R., Margossian, L., Hannon, M., Michaeli, D., Harada, J., Goldberg, R. B. and Fischer, R. L. (1999). Mutations in FIE, a WD polycomb group gene, allow endosperm development without fertilization. Plant Cell 11, 407-415. 
O'Keefe, S. J., Wolfes, H., Kiessling, A. A. and Cooper, G. M. (1989) Microinjection of antisense c-mos oligonucleotides prevents meiosis II in the maturing mouse egg. Proc Natl. Acad. Sci. USA 86, 7038-7042.

Poliakova, T. F. (1964). Development of the male and female gametophytes of Arabidopsis thaliana (L) Heynh. Issledov Genet. USSR 2, 665-667.

Ray, A. (1999). New paradigms in plant embryogenesis: maternal control comes in different flavors. Trends Plant Sci. 3, 325-327.

Redei G. P. (1965). Non-mendelian megagametogenesis in Arabidopsis. Genetics 51, 857-872.

Reiser, L. and Fischer, R. L. (1993). The ovule and the embryo sac. Plant Cell 5, 1291-1301.

Rhoades, M. M. (1956). Genic control of chromosomal behaviour. Maize Genet. Coop. News Lett. 30, 38-48.

Rodkiewicz, B. (1970). Callose in cell walls during megasporogenesis in angiosperms. Planta 93, 39-47.

Schneitz, K., Hulskamp, M. and Pruitt, R. E. (1995). Wild-type ovule development in Arabidopsis thaliana: a light microscope study of cleared whole-mount tissue. Plant J. 7, 731-749.

Schneitz, K., Hulskamp, M., Kopczak, S. D. and Pruitt, R. E. (1997) Dissection of sexual organ ontogenesis: a genetic analysis of ovule development in Arabidopsis thaliana. Development 124, 1367-1376.

Sheridan, W. F., Avalkina, N. A., Shamrov, I. I., Batygina, T. B. and Golubovskaya, I. N. (1996). The macl gene: controlling the commitment to the meiotic pathway in maize. Genetics 142, 1009-1020.

Singleton, W. R. and Mangelsdorf, P. C. (1940). Gametic lethals on the fourth chromosome of maize. Genetics $\mathbf{2 5}, 366-389$.

Somerville, C. R. and Ogren, W. L. (1982) Isolation of photorespiration mutants in Arabidopsis. In Methods in Chloroplast Biology (ed. M Edelman,
R. B. Hallick, and N. H. Chua), pp.129-138. Amsterdam: Elsevier Biomedical Press.

Springer, P. S., McCombie, W. R., Sundaresan, V. and Martienssen, R. A. (1995). Gene trap tagging of PROLIFERA, an essential MCM2-3-5-like gene in Arabidopsis. Science 268, 877-880.

Sundaresan, V., Springer, P., Volpe, T., Haward, S., Jones, J. D. G., Dean, C., Ma, H. and Martienssen, R. (1995). Patterns of gene action in plant development revealed by enhancer trap and gene trap transposable elements. Genes Dev. 9, 1797-1810.

Twell, D., Park, S. K. and Lalanne, E. (1998). Asymmetric division and cell-fate determination in developing pollen. Trends Plant Sci. 3, 305310

Vielle-Calzada, J.-Ph., Thomas, J., Spillane, C., Coluccio, A., Hoeppner, M. A. and Grossniklaus, U. (1999). Maintenance of genomic imprinting at the Arabidopsis medea locus requires zygotic DDMI activity. Genes Dev. (in press).

Webb, M. C. and Gunning, B. E. S. (1990). Embryo sac development in Arabidopsis thaliana. I. Megasporogenesis, including the microtubular cytoskeleton. Sex. Plant Reprod. 3, 244-256.

Willemse, M. T. M. (1981). Polarity during megasporogenesis and megagametogenesis. Phytomorphol. 31, 124-134.

Willemse, M. T. M. and van Went, J. L. (1984). The female gametophyte. In Embryology of Angiosperms (ed. Johri B. M.), pp. 159-196. New York: Springer-Verlag.

Yang, W. C., Ye, D., Xu, J. and Sundaresan, V. (1999). The SPOROCYTELESS gene of Arabidopsis is required for initiation of sporogenesis and encodes a novel nuclear protein. Genes Dev. 13, 21082117 